\title{
Covert presentation of strangulated hiatus hernias after cardiac surgery: A note of caution
}

\author{
Ben Davies, MRCS(Eng), J. Stephen Billing, MA, FRCS(CTh), and Patrick Yiu, PhD, FRCS(CTh), \\ Wolverhampton, United Kingdom
}

Intra-abdominal catastrophes are an uncommon and unpredictable source of morbidity after cardiac surgery. We report the cases of two patients in whom strangulated hiatus hernias developed in the postoperative period and highlight their covert presentations.

\section{CLINICAL SUMMARIES \\ Case 1}

An 81-year-old woman with two-vessel coronary disease and preserved left ventricular function underwent elective off-pump coronary surgery to the posterior descending and left anterior descending coronary arteries. A preoperative chest radiograph was suggestive of hiatus hernia, and the patient took a proton pump inhibitor for dyspepsia. The initial postoperative course was uneventful and she was extubated within 12 hours. A chest X-ray film after chest drain removal showed a distended hiatus hernia, which was subsequently partially decompressed with a nasogastric tube. Over the next 24 hours, respiratory failure, lactic acidosis, and renal failure developed without abdominal symptoms or signs. Transesophageal echocardiography demonstrated good biventricular function and no pericardial collection. White cell count was within normal ranges and the amylase level was mildly raised $(311 \mathrm{U} / \mathrm{mL})$. A computed tomographic scan demonstrated a correctly positioned nasogastric tube and no radiologic evidence of mesenteric strangulation or ischemia (Figure 1). A general surgical opinion was obtained and laparotomy was deemed not indicated. Despite ventilation support, hemofiltration, and later vasoconstrictor support, the patient's condition deteriorated with escalating lactic acidosis. She died of multiorgan failure. At autopsy, an incarcerated sliding hiatus hernia was found with a gangrenous intrathoracic component strangulated around a congenital band between the first part of the duodenum and the gastric fundus.

From the Department of Cardiothoracic Surgery, New Cross Hospital, Wolverhampton, United Kingdom.

Disclosures: None.

Received for publication June 20, 2008; accepted for publication July 6, 2008; available ahead of print Sept 9, 2008.

Address for reprints: Ben Davies, MRCS(Eng), Department of Cardiothoracic Surgery, New Cross Hospital, Wolverhampton, WV10 0QP, United Kingdom (E-mail: ben_davies@doctors.org.uk).

J Thorac Cardiovasc Surg 2010;139:e10-11

0022-5223/\$36.00

Crown Copyright (c) 2010 by The American Association for Thoracic Surgery doi:10.1016/j.jtcvs.2008.07.017

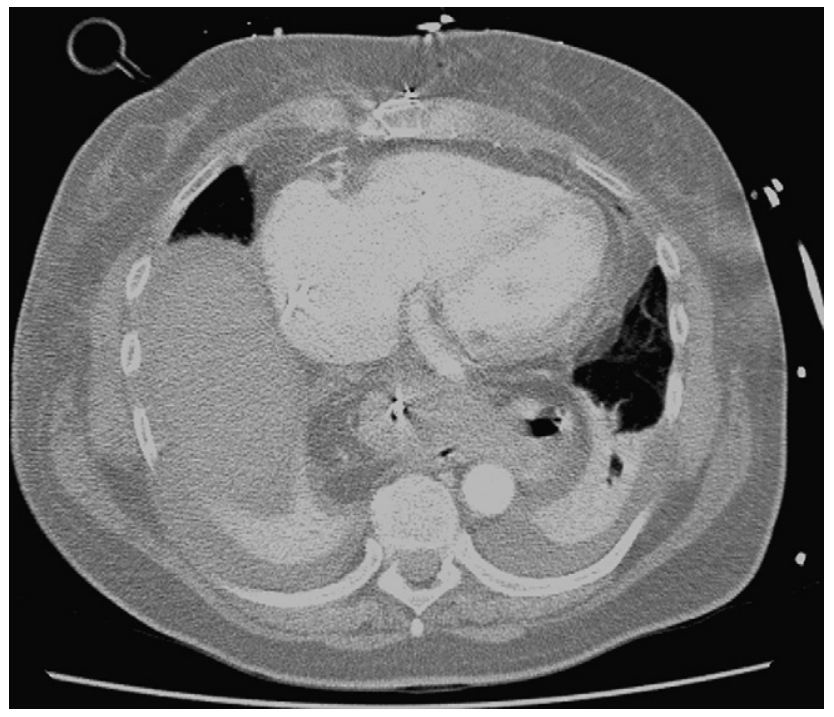

FIGURE 1. Computed tomographic scan showing a correctly positioned nasogastric tube and no radiologic evidence of mesenteric strangulation or ischemia.

\section{Case 2}

A 75-year-old woman with three-vessel disease and poor left ventricular function underwent elective surgical revascularization with cardiopulmonary bypass. A preoperative chest film showed an elevated gas shadow in the left hemithorax. Despite initially good postoperative progress in the first few hours, hemodynamic instability, renal failure, and lactic acidosis developed. A chest radiograph showed a white-out in the left side of the chest (Figure 2). Transesophageal echocardiography demonstrated a fluid collection in the left hemithorax compressing the heart. An intercostal drain was inserted but drained only $100 \mathrm{~mL}$ of serosanguineous fluid, and a repeat radiograph demonstrated an air-fluid level adjacent to the left heart border consistent with a hiatus hernia. A nasogastric tube was introduced but could not be advanced beyond $30 \mathrm{~cm}$, suggestive of a strangulated hernia or gastric volvulus. On re-exploration, a strangulated paraesophageal hernia was found to be causing cardiac tamponade. After reduction, she was returned to the intensive care unit with the sternum splinted open, but she later died of multiorgan failure.

\section{DISCUSSION}

Hiatus hernia and gastroesophaegeal reflux are common in the general population. Complications arising from these 


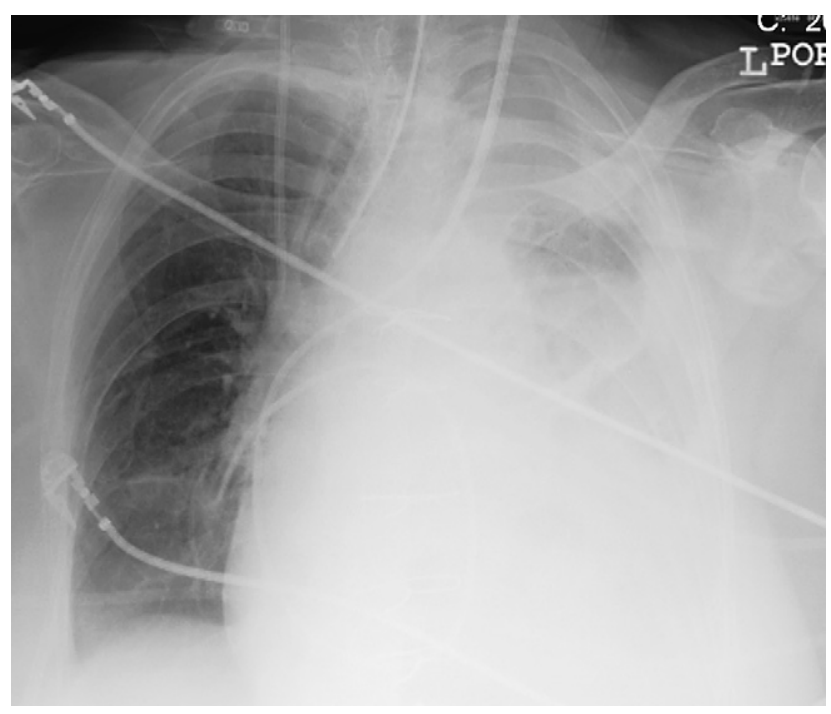

FIGURE 2. Chest radiograph demonstrating white-out in the left hemithorax.

after cardiac surgery are rare. Cardiac compression by a distended intrathoracic stomach, manifesting as reduced cardiac output or arrhythmias, has been described. ${ }^{1,2}$ Both sliding and paraesophageal types of hiatus hernia may strangulate after off-pump and on-pump coronary surgery (patient 1 and patient 2, respectively).We report here two fatalities and comment on lessons learned.

In the first patient, the presentation was more insidious. Diagnosis was made difficult by the paucity of intra-abdominal symptoms and signs. If present, symptoms are likely to be above the diaphragm and can be confused with postoperative sternal pain. The case clearly demonstrated that a normal white cell count and nondiagnostic computed tomographic scan can be erroneously reassuring. Thus, after exclusion of common causes of lactic acidosis, an unresolved etiology of high lactate level should prompt early laparotomy. In the stable patient, upper gastrointestinal endoscopy may aid diagnosis.

The clinical course of the second patient was short and rapid. It too showed that correct diagnosis of a strangulated hiatus hernia can be challenging. Gastric contents in the thorax can resemble a large pleural effusion on chest radiograph and appear confusing on transesophageal echo. ${ }^{3}$ Despite minimal delay to surgical exploration in this case, the patient could not be saved.

Routine placement of nasogastric tubes in patients undergoing cardiac surgery remains debatable. In a randomized trial, Russell and associates ${ }^{4}$ found that nasogastric tubes are not routinely necessary but suggested that certain subgroups, including those with paraesophageal hernia, might benefit from routine placement. After the fatal outcome of our two patients, we believe that the presence of a hiatus hernia (sliding or paraesophageal) identifiable on a preoperative chest x-ray film should mandate prophylactic placement of a largebore nasogastric tube at the time of the operation. The tube should remain in situ for at least 48 hours. This may prevent postoperative distention within the hiatus hernia, which may lead to strangulation. Correct placement once obstruction has occurred may be difficult and also too late.

\section{References}

1. Hunt GS, Gilchrist DM, Hirji MK. Cardiac compression and decompensation due to hiatus hernia. Can J Cardiol. 1996;12:295-6.

2. Devbhandari MP, Khan MA, Hooper TL. Cardiac compression following cardiac surgery due to unrecognised hiatus hernia. Eur J Cardiothorac Surg. 2007;32: 813-5.

3. D'Cruz IA, Feghali N, Gross CM. Echocardiographic manifestations of mediastinal masses compressing or encroaching on the heart. Echocardiography. 1994;11: 523-33.

4. Russell GN, Yam PC, Tran J, Innes P, Thomas SD, Berry PD, et al. Gastroesophageal reflux and tracheobronchial contamination after cardiac surgery: should a nasogastric tube be routine? Anesth Analg. 1996;83:228-32. 